\title{
Cocaine detection by a mid-infrared waveguide integrated with a microfluidic chip
}

\author{
Yu-Chi Chang, ${ }^{* a}$ Philip Wägli, ${ }^{b}$ Vincent Paeder, ${ }^{a}$ Alexandra Homsy, ${ }^{b}$ Lubos Hvozdara, ${ }^{a}$ Peter van der Wal, ${ }^{b}$ \\ Joab Di Francesco, ${ }^{a}$ Nico F. de Rooij ${ }^{b}$ and Hans Peter Herzig ${ }^{a}$
}

Received 25th May 2012, Accepted 25th June 2012

DOI: $10.1039 / \mathrm{c} 2 \mathrm{lc40601b}$

\begin{abstract}
A germanium (Ge) strip waveguide on a silicon ( $\mathrm{Si}$ ) substrate is integrated with a microfluidic chip to detect cocaine in tetrachloroethylene (PCE) solutions. In the evanescent field of the waveguide, cocaine absorbs the light near $5.8 \mu \mathrm{m}$, which is emitted from a quantum cascade laser. This device is ideal for (bio-)chemical sensing applications.
\end{abstract}

Chemical detection and identification based on mid-infrared (midIR) spectroscopy has been widely used for samples in gases, liquids, or solids. In the condensed phase, attenuated total reflection Fourier transform infrared spectroscopy (ATR-FTIR) can conveniently probe the analyte in the evanescent field, but it was often limited to laboratories due to its large footprint. ${ }^{1,2}$ During the last few decades, the progress of mid-IR spectroscopy with mid-IR fiber probes $^{3}$ or planar waveguides has largely reduced the device size and the sample volume. These waveguides are based on silver halides, chalcogenide glasses, GaAs, or hollow waveguides. $^{48}$ In addition, mid-IR quantum cascade lasers (QCLs) provide light sources with high power, high tunability and compact sizes, which can further reduce the size of sensor systems. ${ }^{9}$

Microfluidic systems have been used in combination with IRdetection. The most commonly used techniques are chromatography $^{10-13}$ and electrophoresis ${ }^{10,14,15}$ on a separate microfluidic chip for sample preparation and preconcentration prior to the conventional IR-detection. A microfluidic device integrated with a planar chalcogenide glass waveguide on a silicon substrate was presented for the detection of $\mathrm{N}$-methylaniline at a wavelength of $1.55 \mu \mathrm{m} .{ }^{6}$ Commercial products combining QCLs and flow cells for the detection of oil in water are also available. ${ }^{16}$

Here, we demonstrate a chemical sensor utilizing a mid-IR single-mode strip waveguide in liquid environments. It is integrated with a microfluidic $\operatorname{chip}^{17}$ and evaluated with a QCL at a wavelength of $5.8 \mu \mathrm{m}$. This is the first integration of a Ge strip waveguide with a microfluidic chip and evaluated at a mid-IR wavelength. The laser wavelength is chosen to be at one of the

${ }^{a}$ Ecole Polytechnique Fédérale de Lausanne (EPFL), Institute of Microengineering, Optics \& Photonics Technology Laboratory, Rue A.-L. Breguet 2, Neuchâtel, Switzerland. E-mail: yu-chi.chang@epfl.ch ${ }^{b}$ Ecole Polytechnique Fédérale de Lausanne (EPFL), Institute of Microengineering, Sensors, Actuators and Microsystems Laboratory (SAMLAB), Rue Jaquet-Droz 1, 2000, Neuchâtel, Switzerland absorption peaks of cocaine, ${ }^{18}$ which is our example analyte to demonstrate this technology.

The sensing scheme is illustrated in Fig. 1a, where the light is injected into the waveguide. The analyte in the evanescent field has absorption peaks overlapping with the laser wavelength. The analyte concentration can be determined by measuring the output light from the waveguide.

The mid-IR waveguide is made from a mono-crystalline Ge layer on a $\mathrm{Si}$ substrate with standard photolithography and reactive ion etching by $\mathrm{CF}_{4}$. Undoped $\mathrm{Ge}$ and $\mathrm{Si}$ have low absorption over a wide range of mid-IR wavelengths. This material combination is biocompatible ${ }^{19}$ and compatible with Siprocessing, and can be further integrated with current $\mathrm{Si}$ technology. Fig. 1b shows the cross-section of the strip waveguide. It is single mode for transverse magnetic polarization at a wavelength of $5.8 \mu \mathrm{m}$, while the thickness is $2 \mu \mathrm{m}$ and the width is $2.9 \mu \mathrm{m} .{ }^{20}$ Compared to a slab waveguide, a single mode strip waveguide has a higher fraction of energy in the evanescent field, and hence increases the sensitivity. ${ }^{21}$

The material for the microfluidic system is a UV-curable adhesive NOA81 (Norland Products Inc., Cranbury, NI, USA). Compared to PDMS (polydimethylsiloxane), the most widely used polymeric material for microfluidic devices, this low-cost, commercially available adhesive has better chemical resistance, is impermeable to air and water vapor, and is less prone to swelling upon contact with infrared-transparent organic solvents. ${ }^{17,22}$ Moreover, it shows low adsorption of cocaine (in aqueous or organic solvents), and in proteins (interleukins from blood). ${ }^{23,24}$ Cured

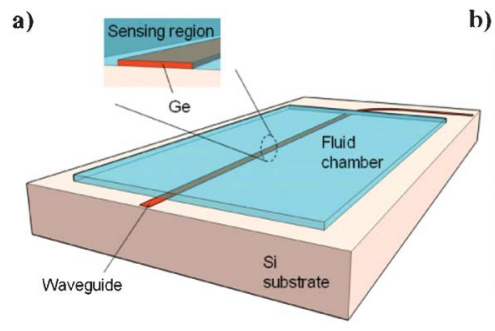

b)

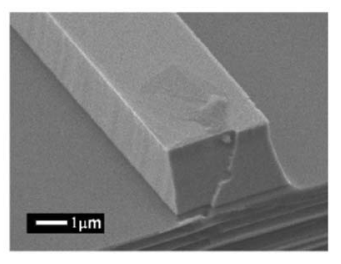

Fig. 1 a) Scheme of the waveguide spectroscopy sensor in liquids: a microfluidic chamber can be bonded onto the substrate. The analyte absorbs light in the evanescent field. b) Image of the cross-section of the Ge waveguide on Si taken with a scanning electron microscope (ref. 20). 
NOA81 has a higher stiffness $(\sim 1 \mathrm{GPa})$ than PDMS. ${ }^{25}$ This allows the building of wide microfluidic channels which do not collapse under the negative pressure used to suck the liquids through the microfluidic chip.

We made the microfluidic system by means of rapid prototyping using NOA81, and by following the fabrication process presented in ref. 16. As shown in Fig. 2, the adhesive was cured on a PDMS master. It was then bonded to the silicon substrate of the $\mathrm{Ge}$ waveguide using oxygen plasma. The adhesive was cured further under the UV-lamp after bonding. The device showed the best adhesion after a high temperature treatment at $130{ }^{\circ} \mathrm{C}$ for $1 \mathrm{~h}$. As shown in Fig. 3, the microfluidic design consists of a simple straight channel ( $1 \mathrm{~mm}$ wide, $7 \mathrm{~mm}$ long and $50 \mu \mathrm{m}$ deep) with an inlet and an outlet. After use, the low-cost, disposable, polymeric microfluidic system can be detached from the substrate by soaking it in acetone and the waveguide can be recycled.

Cocaine detection is a pilot demonstration for this device. The wavelength of the QCL is $5.8 \mu \mathrm{m}$, which overlaps with the specific absorption peak of cocaine. We dissolved cocaine (purity $>98 \%$, Lipomed AG Switzerland, purchased with permission of the Cantonal Drug Administration of Neuchâtel) in PCE, which has high transmission in a large mid-IR band.

The measurement setup consists of an optical system measuring the waveguide output, and a syringe pump system for liquid sample handling. In Fig. 4, the light emitted from the QCL was coupled with the strip waveguide by a $1 / f \mathrm{ZnSe}$ doublet. After interaction with the sample in the liquid chamber, the light went through a waveguide bend and was redirected to an MCT (mercury cadmium telluride) detector by two parabolic mirrors. The use of a bent waveguide avoided stray light on the emission axis of the QCL and increased the signal to noise ratio.

A syringe pump connected to the outlet of the microfluidic system sucked the liquid through the device. The multi-selection valve at the inlet enabled switching between reservoirs of pure PCE solvent and reservoirs with different concentrations of cocaine dissolved in PCE.

Before the measurement of cocaine, the device was tested using a waveguide without a microfluidic chip. We put a water drop on the substrate and controlled its size dynamically with a pipette. Since water has strong absorption near this wavelength, when the water drop covered the waveguide by different lengths, the light transmission of the waveguide changed simultaneously. Fig. 5 shows the real time measurement with 5 different water-covered lengths. The response time is about $0.5 \mathrm{~s}$.
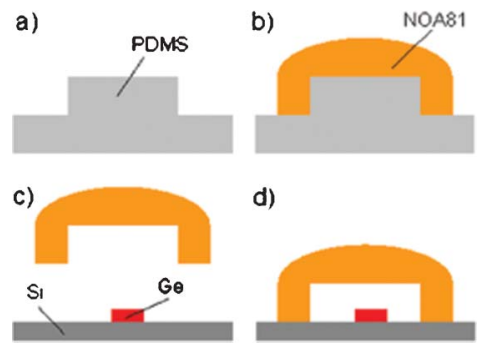

d)

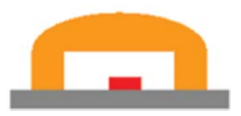

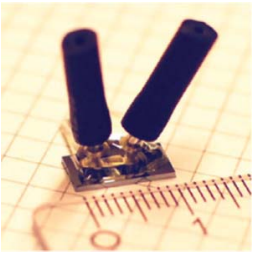

Fig. 3 Image of the Ge waveguide bonded to the microfluidic channel: the microfluidic channel is $1 \mathrm{~mm}$ wide, $7 \mathrm{~mm}$ long, and $50 \mu \mathrm{m}$ deep.

For cocaine measurement, we used a waveguide integrated with a microfluidic chip. The result is shown in Fig. 6, in which the waveguide output light intensity was measured when we switched different concentrations of cocaine in real time. The flow rate was $50 \mu \mathrm{min}^{-1}$. Between each concentration, we added pure PCE solvent at the same flow rate for 5-7 $\mathrm{min}$. The device responded well to each switch of concentration. The transmission drop was linearly proportional to the cocaine concentration, while the lowest detected concentration was $100 \mu \mathrm{g} \mathrm{ml}^{-1}$. The detection limit can be further improved by reducing the system noise and using a longer waveguide. The shift of the average output-power is due to the fluctuation of alignment between the laser and the waveguide.

The slow response to the switch of concentrations was due to the length of the tubing between the device and the fluidic valve. This is tested by using a long $(\sim 20 \mathrm{~cm})$ and a short $(\sim 6 \mathrm{~cm})$ tube for measuring the switch from pure PCE to a cocaine concentration of $100 \mu \mathrm{g} \mathrm{ml}^{-1}$. Fig. 7 shows the response times with the two tubes, and the shorter tube responds about twice as fast as the longer one. The response time is the time taken for the transmission value to change between $10 \%$ and $90 \%$ of the difference between the mean transmission values of pure PCE and $100 \mu \mathrm{g} \mathrm{ml}^{-1}$ cocaine (as shown in Fig. 7).

Cocaine detection in human saliva is a potential application and also a goal of this study. In parallel to the presented device, we are developing an integrable microfluidic system for efficient liquidliquid extraction of the analyte cocaine from saliva to PCE. ${ }^{18,26}$

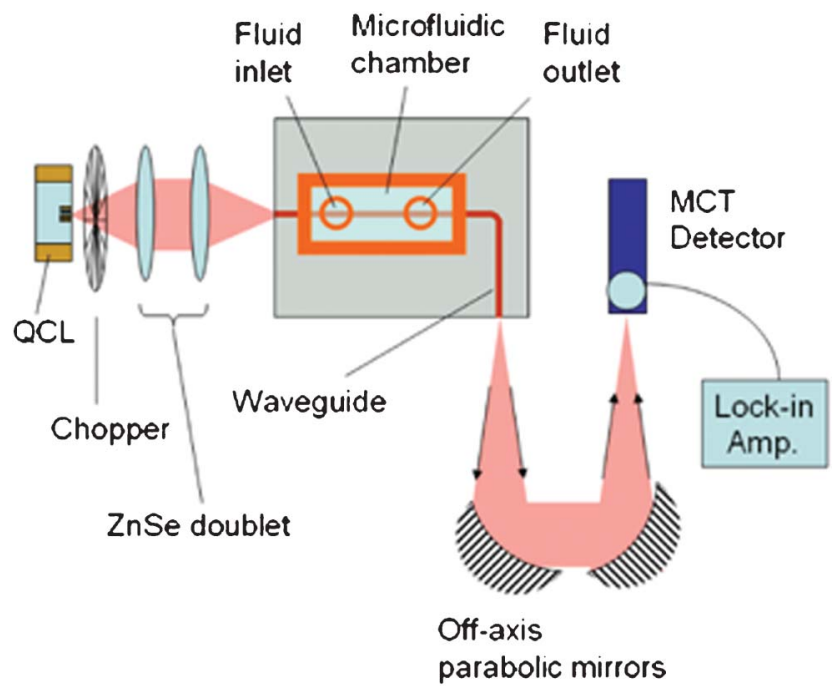

Fig. 4 Measurement setup: the waveguide is coupled with the QCL by a ZnSe doublet and to a MCT detector by two parabolic mirrors. Lightliquid interaction happens in the microfluidic chamber in which cocaine solutions with different concentrations keep a constant flow. 


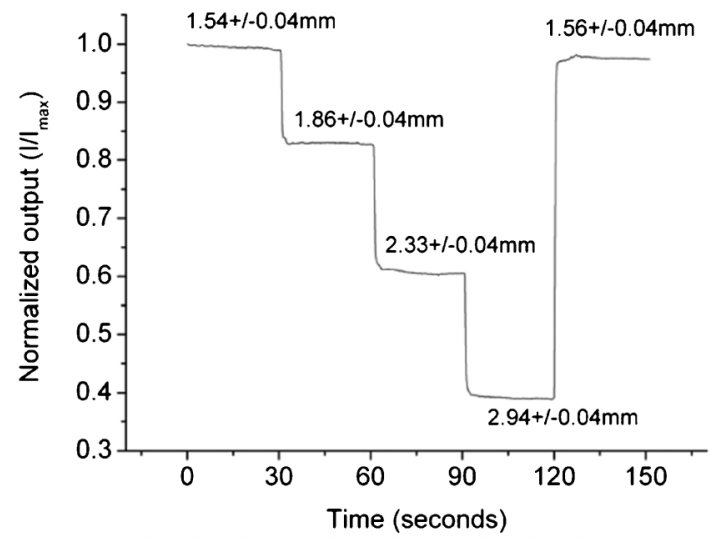

Fig. 5 Water drop test without a microfluidic chip: the water drop covered different lengths of the waveguide when we changed the drop-size in real time. The waveguide transmission showed a fast response.

This sample pretreatment allows analyte preconcentration and avoids the problem of light absorption in the water. As the peak value of cocaine concentration in saliva can be as high as $500 \mu \mathrm{g}$ $\mathrm{ml}^{-1},{ }^{18}$ the current detection limit of this device can be improved to a practical level. The signal to noise ratio of this device is now mainly limited by the alignment fluctuation of the laser. An integrated splitter and a reference waveguide can eliminate this noise and a large improvement can be expected. Stabilized temperature and mechanical vibration can also reduce the noise. Further improvement includes the use of a long waveguide, which can be supported by the high output power of the QCL and the low loss of Ge waveguides. ${ }^{20}$ The coupling efficiency of the laser is currently around $9 \%$, and can be improved 5-10 fold with butt coupling method.

This device demonstrated the integration of a mid-IR Ge waveguide with a microfluidic system. Cocaine detection is a pilot example of this technology. The detection limit of this device still needs optimization when it is compared with other optical methods which have detection limits in the range of several $\mathrm{ng} \mathrm{ml}{ }^{-1}$ to several $\mu \mathrm{g} \mathrm{ml}^{-1}{ }^{18}$ but this device has the advantage of the ability to obtain spectral information if a tunable QCL is used. The compact size of the QCL and the waveguides allows the design of portable systems. In addition, this real time experiment

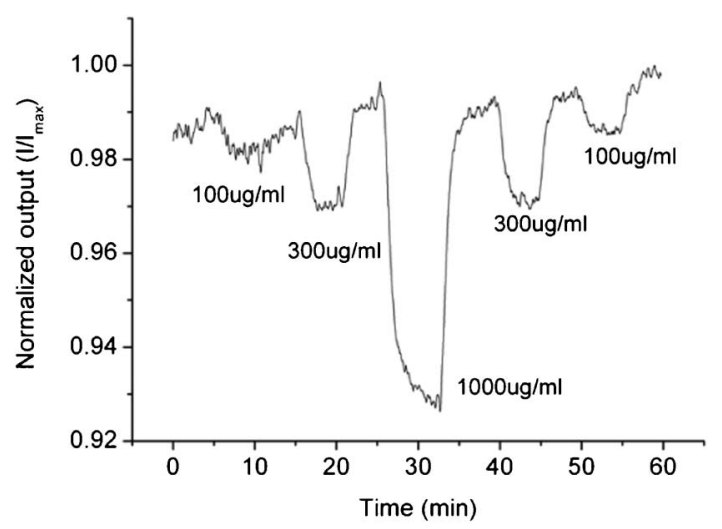

Fig. 6 Waveguide output with different cocaine concentrations: in the dynamic measurement, the smallest detected concentration was $100 \mu \mathrm{g} \mathrm{ml}^{-1}$.

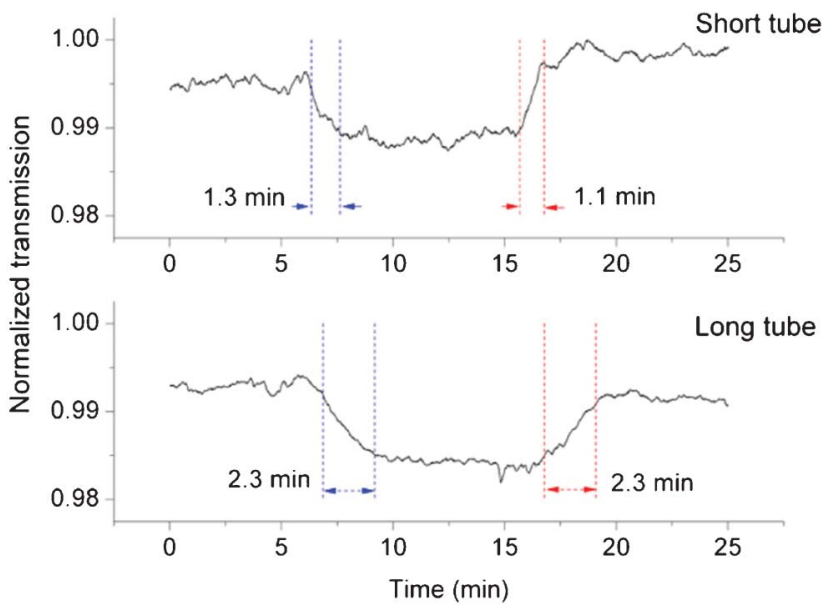

Fig. 7 Response time: it is related to the tubing length between the fluidic valve and the device. Between pure PCE and $100 \mu \mathrm{g} \mathrm{ml}^{-1}$ cocaine in PCE solution, the shorter tube showed a faster response time.

shows the capability of this device for in-line measurement and studying dynamic phenomena in liquids. The integrated microfluidics enables further developments of on-chip sample handling and treatment before or after the optical sensing.

This project is scientifically evaluated by SNSF, financed by the Swiss Confederation and funded by Nano-Tera.

\section{References}

1 N. Harrick, Phys. Rev. Lett., 1960, 4, 224-226.

2 J. Fahrenfort, Spectrochim. Acta, 1961, 17, 698-709.

3 A. C. McIntyre, M. L. Bilyk, A. Nordon, G. Colquhoun and D. Littlejohn, Anal. Chim. Acta, 2011, 690, 228-233.

4 L. Hvozdara, S. Gianordoli, G. Strasser, W. Schrenk, K. Unterrainer, E. Gornik, C. S. S. S. Murthy, M. Kraft, V. Pustogow, B. Mizaikoff, A. Inberg and N. Croitoru, Appl. Opt., 2000, 39, 6926.

5 O. Eyal, V. Scharf, S. Shalem and A. Katzir, Opt. Lett., 1996, 21, 1147.

6 J. Hu, V. Tarasov, A. Agarwal, L. Kimerling, N. Carlie, L. Petit and K. Richardson, Opt. Express, 2007, 15, 2307-2314.

7 C. Charlton, M. Giovannini, J. Faist and B. Mizaikoff, Anal. Chem., 2006, 78, $4224-4227$.

8 Kim Seong-Soo, Christina Young and Boris Mizaikoff, Anal. Bioanal. Chem., 2008, 390, 231-237.

9 J. Faist, F. Capasso, D. L. Sivco, C. Sirtori, A. L. Hutchinson and A. Y. Cho, Science, 1994, 264, 553-556.

10 J. Kuligowski, G. Quintás, M. de la Guardia and B. Lendl, Anal. Chim. Acta, 2010, 679, 31-42.

11 M. Haberkorn, J. Frank, M. Harasek, J. Nilsson, T. Laurell and B. Lendl, Appl. Spectrosc., 2002, 56, 902-908.

12 A. Edelmann, J. Diewok, J. R. Baena and B. Lendl, Anal Bioanal Chem, 2003, 376, 92-97.

13 B. Lendl, J. Frank, R. Schindler, A. Müller, M. Beck and J. Faist, Anal. Chem., 2000, 72, 1645-1648.

14 S. Kulka, G. Quintas and B. Lendl, Analyst, 2006, 131, 739-744.

15 S. Kulka, G. Quintas and B. Lendl, Vib. Spectrosc., 2006, 42, 392-396.

$16 \mathrm{http}: / /$ www.quantared.com.

17 P. Wägli, A. Homsy and N.F. de Rooij, Sens. Actuators, B, 2011, 156(2), 994-1001.

18 K. M.-C. Hans, M. Gianella and M. W. Sigrist, in Proc. SPIE 8229, 2012, 822919-1-822919-7.

19 J. Fan and P. K. Chu, Small, 2012, 6, 2080-2098.

20 Y.-C. Chang, V. Paeder, L. Hvozdara, J.-M. Hartmann and H. P. Herzig, Optics Letters, 2012, 37(14), 2883-2885.

21 X. Wang, S.-S. Kim, R. Roßbach, M. Jetter, P. Michler and B. Mizaikoff, Analyst, 2012, 137, 2322.

22 L.-H. Hung, R. Lin and A. P. Lee, Lab Chip, 2008, 8, 983. 
23 A. Homsy, P. D. van der Wal, W. Doll, R. Schaller, S. Korsatko, M. Ratzer, M. Ellmerer, T. R. Pieber, A. Nicol and N. F. de Rooij, Biomicrofluidics, 2012, 6, 012804.

24 P. Wägli, A. Homsy and N. F. de Rooij, International Meeting on Chemical Sensors (IMCS), Nürnberg, Germany, 2012.
25 D. Bartolo, G. Degré, P. Nghe and V. Studer, Lab Chip, 2008, 8, 274

26 P. Wägli, Y.-C. Chang, P. D. van der Wal, L. Hvozdara, A. Homsy, H. P. Herzig, N. F. de Rooij, Droplet-based liquid-liquid extraction and on-chip $I R$-waveguide-spectroscopy detection of cocaine in human saliva, accepted for oral presentation at $\mu$ TAS-Conference 2012, Okinawa, Japan. 